April, 1994

\title{
Towards exact bosonization of the Calogero-Sutherland model
}

\author{
D.V.Khveshchenko \\ Physics Department, Princeton University, \\ Princeton, NJ 08544 \\ and Landau Institute for Theoretical Physics, \\ 2, st.Kosygina, 117940, Moscow, Russia
}

\begin{abstract}
We demonstrate that the exact form-factors of the Calogero-Sutherland model which were recently found in [10] in confirmation of the congectures earlier made by Haldane (Ref. [9]) can be reproduced in the framework of some bosonization procedure in momentum space. This observation implies a possibility of an exact bosonization of this model describing one-dimensional anyons.
\end{abstract}


The Calogero-Sutherland (CS) model [1] of one-dimensional (1D) spinless particles with long-ranged $1 / x^{2}$ interaction $\left(V(x)=\frac{\pi^{2} \lambda(\lambda-1)}{L^{2}\left(\sin \frac{\pi x}{L}\right)^{2}}\right.$ in a finite size $L$ system) provides a remarkable example of an exactly solvable system which can be viewed as a continuous deformation away from either ideal Fermi or Bose gas.

At special values $\lambda=1 / 2,1$ and 2 the original continuous model describes statistics of eigenvalues of random matrices belonging to one of the three Dyson's ensembles. Recently these special cases were intensively studied in the framework of the theory of universal spectral correlations in random systems [2]. The lattice version of the CS model at $\lambda=2$ also induced a formulation of the Haldane-Shastry spin chain model [3] possessing rich Yangian symmetry in the case of a finite length chain [4]. Further generalizations introducing particles with internal degrees of freedom in both continuous and lattice versions led to a family of new solvable models with graded $S U(m \mid n)$ symmetries [5].

The spectrum of the CS model obtainable by means of the so-called Asymptotic Bethe Ansatz (ABA) [6] appears to be strikingly simple and enables one to consider this system as an ideal gas of excitations obeying fractional exclusion principle formulated in [7]. The latter was originally proposed as an alternative definition of fractional quantum statistics which is independent of the space dimension in contrast to the conventional notion of exchange statistics based on braiding relations for corresponding operators [ [8]

Remarkably, in the framework of the CS model these two definitions of fractional statistics demonstrate their perfect consistency. In its fractional exclusion formulation the principle of fractional statistics manifests itself as a set of rules governing allowed patterns of occupation numbers of states with momenta $k_{i}$ obeying ABA equations [6] for a system with periodic boundary conditions

$$
L k_{i}=2 \pi n_{i}+\pi(\lambda-1) \sum_{j \neq i} \operatorname{sgn}\left(k_{i}-k_{j}\right)
$$

Eigenvalues of the Hamiltonian and the total momentum operator are given by free particle's expressions: $E=\frac{1}{2 m} \sum_{i} k_{i}^{2}$ and $P=\sum_{i} k_{i}$. In the case of a rational coupling constant $\lambda=\frac{M}{N}$ the ground state $\left(z_{i}=e^{\frac{2 \pi i x_{i}}{L}}\right)$ 


$$
\Psi_{0}\left(\left\{z_{i}\right\}\right)=\prod_{i<j}^{\mathcal{N}}\left(z_{i}-z_{j}\right)^{\lambda} \prod_{i}^{\mathcal{N}} z_{i}^{-\lambda \frac{\mathcal{N}-1}{2}}
$$

can be characterised as a periodic array of one occupied and $M-1$ empty states associated with consequent non-negative integers $n_{i}$. Patterns describing excited states are also subjected to a very restrictive selection rule which dictates that one can only move $N$ momenta out of the ground state distribution simultaneously. This rule can be naturally interpreted in terms of fundamental quasiparticles with disperion $\epsilon_{q}(u)=\frac{1}{2}\left(u^{2}-k_{F}^{2}\right),\left(|u|>k_{F}=\pi \rho\right.$ where $\rho$ is a density of particles of mass $m=1)$ and quasiholes $\left(\epsilon_{h}(v)=\frac{1}{2 \lambda}\left(k_{F}^{2}-v^{2}\right),|v|<k_{F}\right)$ as a statement that an elementary excitation at conserved particle number is constituted by $N$ quasiparticles and $M$ quasiholes. On the basis of this constraint it was first anticipated in 99 and then explicitly found in [10] that dynamical correlation functions of local operators such as a fundamental field operator $\Psi(x)$ and density $\rho(x)$ have exclusively simple structure. It stems from the fact that sums over intermediate states $\mid \nu>$ in formal expansions (where $O=\Psi, \rho)$

$$
<v a c|O(x, t) O(0,0)| v a c>=\sum_{\nu}|<\nu| O(0,0)|v a c>|^{2} \exp \left(i E_{\nu} t-i P_{\nu} x\right)
$$

now amount to single terms given by the only nonzero form-factors

$$
\left.<u_{1}, \ldots u_{N-1} ; v_{1}, \ldots v_{M}|\Psi(0)| v a c\right\rangle=C_{M, N-1} \frac{\prod_{i<j}^{M}\left(v_{i}-v_{j}\right)^{1 / \lambda} \prod_{i<j}^{N-1}\left(u_{i}-u_{j}\right)^{\lambda}}{\prod_{i}^{M}\left(1-v_{i}^{2}\right)^{\frac{1-1 / \lambda}{2}} \prod_{i}^{N-1}\left(u_{i}^{2}-1\right)^{\frac{1-\lambda}{2}} \prod_{i, j}^{M(N-1)}\left(u_{i}-v_{j}\right)}
$$

and

$$
<u_{1}, \ldots u_{N} ; v_{1}, \ldots v_{M}|\rho(0)| v a c>=C_{M, N} \frac{\prod_{i<j}^{M}\left(v_{i}-v_{j}\right)^{1 / \lambda} \prod_{i<j}^{N}\left(u_{i}-u_{j}\right)^{\lambda}\left(\sum_{i}^{N} u_{i}-\frac{1}{\lambda} \sum_{i}^{M} v_{i}\right)}{\prod_{i}^{N}\left(u_{i}^{2}-1\right)^{\frac{1-\lambda}{2}} \prod_{i}^{N}\left(1-v_{i}^{2}\right)^{\frac{1-1 / \lambda}{2}} \prod_{i, j}^{N M}\left(u_{i}-v_{j}\right)}
$$

where $C_{n m}$ are known normalization factors [9], [10] and rapidities are normalized with respect to the Fermi momentum $\left(k_{i} \rightarrow k_{i} / k_{F}\right)$.

Formulae (4) for $\lambda=2$ [1] and (5) for $\lambda=2,1$ and $1 / 2$ [12] were first obtained by means of supermatrix model calculus. In [10] they were found at arbitrary rational $\lambda$ by using 
the method of Jack polynomials which was developed in [13] to compute static correlations functions related to Selberg integrals.

This basically combinatoric approach explicitly implements the fractional exclusion principle. On the other hand, in view of the abovementioned equivalence of two definitions of fractional statistics one might expect that the problem also allows a purely algebraic solution based on monodromy properties of quasiparticle field operators and their braiding relations.

Indeed, one might be tempted to interpret formulae (4)and (5) as vacuum expectation values of some "vertex operators" constructed from a free boson field ( 2D Coulomb gas correlators). In this Letter we present arguments in favor of such an interpretation which should be actually viewed as a possibility to carry out an exact bosonization of the CS model.

In its original formulation the conventional bosonization procedure was developed to describe longwavelength properties of gapless 1D fermions with contact interactions in terms of their collective excitations (charge and spin density waves). The procedure involves a coordinate space construction of the fundamental fermion field operator in terms of bosons $\left(\psi(x) \sim \sum_{R, L} e^{i \phi_{R, L}(x) \pm i k_{F} x}\right)$ which is suitable only for a description of low-energy fermionic excitations with linear dispersion in the vicinity of well separated Fermi points. Apparently, intending to develop an exact bosonization scheme valid at all scales one can no longer use this simple representation.

Conceivably, some hints for a proper construction in coordinate space can be inferred from the fact that the wave function of the ground state (2) (as well as lowest excited states) can be reproduced as vacuum expectation values of a product of $\mathcal{N}$ "quasi-chiral" vertex operators $\psi(z)=e^{i \sqrt{\lambda} \phi(z)}$ written in terms of some formal bosonic variable

$$
\Psi_{0}\left(\left\{z_{i}\right\}\right)=<\prod_{i}^{\mathcal{N}} e^{i \sqrt{\lambda} \phi\left(z_{i}\right)} e^{-i \frac{\lambda}{2}(\mathcal{N}-1) \phi(0)}>
$$

where the gaussian average has to be taken as $<\phi(z) \phi\left(z^{\prime}\right)>=-\ln \left(z-z^{\prime}\right)$. Analogously, the wave function $\Psi(z)=\Psi_{0}(z) \prod_{i}^{\mathcal{N}} \prod_{j}^{\mathcal{N}_{h}}\left(z_{i}-\zeta_{j}\right)$ corresponding to the state with $\mathcal{N}_{h}$ quasiholes can be readily obtained by inserting a product of $\mathcal{N}_{h}$ operators $e^{\frac{i}{\sqrt{\lambda}} \phi(\zeta)}$ into (6). 
These observation are equivalent to those made about Laughlin trial wave functions [14] which are nothing but a result of analytic continuation of the CS model wave functions defined on a unit circle into an entire complex $z$ plane (if nonanalytic gaussian factors $e^{-\left|z_{i}\right|^{2} / 4}$ are, as usual, asigned to integration measure).

To make this heuristic construction physically meaningful we implement another observation from the so-called collective field theory of unitary matrix models [15]. It was shown in 15$]$ that the system of free nonrelativistic fermions is equivalent to the cubic Hamiltonian

$$
H=\frac{\rho}{2}\left((\partial \varphi)^{2}+(\partial \theta)^{2}\right)+\frac{1}{2}(\partial \varphi)^{2} \partial \theta+\frac{1}{6}(\partial \varphi)^{3}
$$

where bosonic variables $\varphi(x)$ and $\theta(x)$ are conjugated to each other: $[\varphi(x), \theta(y)]=$ $\frac{i \pi}{2} \operatorname{sgn}(x-y)$ and related to local particle number $\left(\rho=\frac{1}{\pi} \partial \varphi\right)$ and current $\left(j=\frac{1}{\pi} \partial \theta\right)$ densities. Elaborating on this theory one can see that although time evolution of bosonic variables is quite complicated, it turns out that some exponential composite operators $W_{a b}=\exp (i a \varphi+i b \theta)$ may have much simpler dynamics. In particular, the exponentials $W_{1,1}=\exp (i \varphi+i \theta)$ satisfies the equation of motion for free fermions.

Moreover the exponentials $W_{\lambda, 1}=\exp (i \lambda \varphi+i \theta)$ obey the equation of motion of the CS model at arbitrary $\lambda$ (modulo some normal ordering ambiguities). We believe that this circumstance makes it possible to consider $W_{\lambda, 1}$ as a plausible representation of the fundamental CS field with time dependence resulting from the evolution of variables $\varphi(x)$ and $\theta(x)$ determined by the cubic Hamiltonian (7). It implies that the "quasi-chiral" boson field appearing in (6) can be identified as $\phi(z)=\lambda \varphi+\theta$.

Referring to $W_{1,1}$ as a bare fermion $\psi(x)$ one can also treat $W_{\lambda, 1}$ as a "dressed" anyon field operator:

$$
\tilde{\psi}(x)=e^{i(\lambda-1) \pi \int_{-\infty}^{x} \psi^{\dagger}(y) \psi(y) d y} \psi(x)
$$

which is another formulation of the Eq.(1). In terms of $\tilde{\psi}(x)$ the CS Hamiltonian becomes quadratic which sheds additional light on the remarkable simplicity of the spectrum of the CS model describing purely statistical interaction. 
However this real space bosonization approach doesn't provide an immediate construction of eigenstates of the model. Even for the case $\lambda=1$ studied in the context of the $1+1$ dimensional string theory in 16$]$ it appears to be quite involved.

On the other hand, this aim can be readily achieved in the framework of a dual approach of "momentum space bosonization" recently proposed in [17]. Being applied to the Sine-Gordon model at $\beta=1$ this method provided a bosonic representation of a massive relativistic fermion field which facilitates computations of formfactors of other operators built from the Sine-Gordon field. This study also clarified a physical origin of the bosonic field $\phi(z)$ from (6) as a generating function for the integrals of motion $I_{n}=\sum_{i} p_{i}^{n}$ of the free fermion system

$$
\phi(z)=\sum_{n \neq 0} \frac{I_{n}}{i n}
$$

where $I_{n}$ at $n<0$ are "angle" variables conjugated to those at $n>0$ ("action" variables). Altogether they form a $U(1)$ Kac-Moody algebra: $\left[I_{n}, I_{m}\right]=n \delta_{n m}$.

Being formulated this way the method of [17] allows a straightforward generalization onto the case of interacting systems which are characterised by factorizable scattering and possess $\mathcal{N}$ integrals of motion given by powers of some Lax operator: $I_{n}=\operatorname{Tr} L^{n}$. Since the CS model determined by the Lax operator $L_{i j}=\delta_{i j} p_{i}+\frac{\pi \lambda}{L}\left(1-\delta_{i j}\right) \cot \frac{\pi}{L}\left(x_{i}-x_{j}\right)$ allows the ABA solution, the eigenvalues of integrals of motion can be also presented in the free particle form $I_{n}\left|\left\{k_{i}\right\}>=\sum_{i} k_{i}^{n}\right|\left\{k_{i}\right\}>$ where $k_{i}$ are asymptotic momenta obtainable from (6).

In the picture of second quantization the integrals of motion acquire free particle form in terms of quasiparticle and quasihole creation-annihilation operators $Z_{a}(u)(a=p, h)$ labeled by asymptotic momenta. Being considered on general grounds operators $Z_{a}$ obey Zamolodchikov-Faddeev (ZF) algebra $(a=1, \ldots, f)$ [18:

$$
\begin{array}{r}
Z_{a}(u) Z_{b}(v)=S_{a b}(u-v) Z_{b}(v) Z_{a}(u) \\
Z_{a}^{\dagger}(u) Z_{b}^{\dagger}(v)=S_{a b}(u-v) Z_{b}^{\dagger}(v) Z_{a}^{\dagger}(u) \\
Z_{a}(u) Z_{b}^{\dagger}(v)=S_{a b}^{-1}(u-v) Z_{b}^{\dagger}(v) Z_{a}(u)+\delta_{a b} \delta(u-v)
\end{array}
$$


where $S_{a b}(u-v)$ is a scattering matrix of excitations which depends on relative rapidity. In the case $S_{a b}(u)=\gamma_{a} \gamma_{b} S(u)\left(\gamma_{a}\right.$ is a $U(1)$ "charge" of a species a) bosonization procedure can be performed by constructing a representation of the algebra $(10) Z_{a}(u)=e^{i \gamma_{a} \Phi(u)}$ in terms of a one-component bosonic field with commutation relations

$$
[\Phi(u), \Phi(v)]=\ln S(u-v)
$$

which infer the vacuum expectation value

$$
<0|\Phi(u) \Phi(v)| 0>=-\ln f(u-v)
$$

with $f(u)$ determined by the equation $S(u)=f(-u) / f(u)$. In terms of ZF operators the integrals of motion can be written as

$$
I_{n}=\sum_{a}^{f} \int_{\Lambda_{a}} d u Z_{a}^{\dagger}(u) p_{a}^{n}(u) Z_{a}(u)
$$

where $\Lambda_{a}$ is a region of rapidities supporting excitations $Z_{a}$ with momenta $p_{a}(u)$.

Expressions for local (in momentum space) operators bilinear in $Z_{a}$ can be found in a manner similar to the point-splitting procedure in the conventional real space bosonization which yields

$$
Z_{a}^{\dagger}(u) Z_{a}(u)=\frac{\gamma_{a}}{\pi} \frac{\partial \Phi(u)}{\partial u}
$$

With the use of (14) the bosonic phase (9) providing a real space representation of the fundamental field operator $\psi(z)$ can be recovered in the form

$$
\phi(z)=\sum_{a}^{f} \frac{\gamma_{a}}{2} \int_{\Gamma_{a}} \frac{d u}{2 \pi i} \ln \left(1-u^{2} z^{2}\right) \frac{\partial \Phi(u)}{\partial u}
$$

where integrals in the complex plane of rapidities are taken over contours $\Gamma_{a}$ encircling real axis intervals $\Lambda_{a}$.

In the particular case of the CS model scattering matrix is given by matrix elements $S_{p p}\left(u_{1}-u_{2}\right)=\pi \lambda \operatorname{sgn}\left(u_{1}-u_{2}\right), S_{h h}\left(v_{1}-v_{2}\right)=\frac{\pi}{\lambda} \operatorname{sgn}\left(v_{1}-v_{2}\right)$ and $S_{p h}(u-v)=\pi \operatorname{sgn}(u-v)$ equal to statistical phase factors assigned to elementary excitations having $\gamma_{p, h}= \pm \lambda^{ \pm 1 / 2}$ with rapidities equal to their velocities. It then leads to ZF operators 


$$
Z_{p}(u)=e^{i \sqrt{\lambda} \Phi(u)}, \quad Z_{h}(v)=e^{-i \frac{\Phi(v)}{\sqrt{\lambda}}}
$$

creating eigenstates $\left(\left|u_{i} ; v_{j}>=\prod_{i} Z_{p}^{\dagger}\left(u_{i}\right) \prod_{j} Z_{h}^{\dagger}\left(v_{j}\right)\right| v a c>\right)$ and $<0|\Phi(u) \Phi(v)| 0>=-\ln (u-$ $v)$. By construction $\Phi(u)$ is subjected to the condition $\Phi(0)=0$ and has no singularities on the real axis. Naturally, $\Phi(u)$ should also vanish at $|u| \rightarrow \infty$. Although general rules of choosing integration contours in (15) remain to be clarified we believe that a correct prescription is to take contour intergals at positive and negative Reu separately to keep a distinction between left and right moving excitations.

Then integrals taken over four contours encircling intervals $(-\infty,-1],[-1,0),(0,1]$, and $[1, \infty)$ yield the following expression for the fundamental CS field operator at $z=1(x=0)$ :

$$
\psi(0) \sim \exp \frac{i}{2}\left(\sqrt{\lambda}-\frac{1}{\sqrt{\lambda}}\right)(\Phi(1)+\Phi(-1))
$$

Together with the formulae for $Z_{p}(u)$ and $Z_{h}(v)$ given by (16) the gaussian average

$$
<u_{1}, \ldots u_{N-1} ; v_{1}, \ldots v_{M}|\psi(0)| v a c>=<\prod_{i}^{N-1} Z_{p}\left(u_{i}\right) \prod_{j}^{M} Z_{h}\left(v_{j}\right) \psi(0)>
$$

reproduces the form- factor (4) up to a normalization constant. The abovementioned selection rules constraining allowed quasiparticle contents of asymptotic states provide that (18) is free of infrared divergent factors potentially coming out of averages $\left\langle\Phi^{2}(u)>\right.$ at coincident momenta.

Notice that one can only take the free fermion limit $(M=N=1)$ in the final formula (4) to obtain $<v|\psi(0)| v a c>=\theta(1-|v|)$.

By inspection it is also easy to see that to reproduce the form-factor of the density operator (5) one can use the following prescription

$$
\rho(0) \sim P \exp \frac{i}{2}\left(\sqrt{\lambda}-\frac{1}{\sqrt{\lambda}}\right)(\Phi(1)+\Phi(-1))
$$

where $P=\sum_{i}^{N} u_{i}-\frac{1}{\lambda} \sum_{j}^{M} v_{j}$ is a total momentum of excitations which can be identified with $\left.\left(\partial_{z} \phi(z)-\bar{\partial}_{\bar{z}} \phi(\bar{z})\right)\right|_{z \rightarrow 1}$. The relation (19) should result from an operator algebra of $\psi(z)$ and $\psi^{\dagger}(z)$ at arbitrary $z$ which remains to be established. 
In conclusion we notice that a systematic approach to a complete bosonization of the CS model could be built on the basis of its infinite symmetry recognised as $W_{\infty}$ [19]. The corresponding linear algebra is formed by operators $W_{n}^{s}=\sum_{i}^{\mathcal{N}} x_{i}^{s-1} p_{j}^{n+s-1}+\ldots$ which can be recursively obtained as $W_{n}^{s}=\frac{1}{2(n+s)}\left[\sum_{i}^{\mathcal{N}} x_{i}^{2}, W_{n+2}^{s-1}\right]$ from the CS integrals of motion: $W_{n}^{1}=I_{n}$. In particular, the CS Hamiltonian is among these operators $\left(H_{C S}=W_{2}^{1}\right)$. Representation theory of $W_{\infty}$ in terms of free bosons discussed in [20] might appear to be useful here.

A complete CS model bosonization scheme valid at all scales would be an important step towards an exact formulation of a (nonlinear) hydrodynamics of 1D fluids of interacting fermions.

The author is indebted to F.D.M.Haldane for valuable discussions and for the opportunity to become aware of Refs. [9] and [10] prior to publication. This work was supported by the NSF Grant. 


\section{REFERENCES}

[1] F.Calogero, J.Math.Phys.10, 2191 (1969); B.Sutherland, Phys.Rev.A4, 2019 (1971); ibid A5, 1372 (1972).

[2] B.D.Simons, P.A.Lee, and B.L.Altshuler, Phys.Rev.Lett.70, 4122 (1993).

[3] F.D.M.Haldane, Phys.Rev.Lett.60, 635 (1988); B.S.Shastry, Phys.Rev.Lett.60, 639 (1988).

[4] F.D.M.Haldane, Z.N.C.Ha, J.C.Talstra, D.Bernard, and V.Pasquier, Phys.Rev.Lett.69, 2021 (1992).

[5] Z.N.C.Ha and F.D.M.Haldane, Phys.Rev.B46, 9359 (1992);

N.Kawakami, Phys.Rev.B46, 1005 (1992); Phys.Rev.Lett.71, 275 (1993).

[6] B.Sutherland, Lacture Notes in Physics, 242, Springer-Verlag, 1985.

[7] F.D.M.Haldane, Phys.Rev.Lett.67, 937 (1991).

[8] "Fractional Statistics and Anyon Superconductivity", ed. FF.Wilczek, World Scientific (Singapore), 1990.

[9] F.D.M.Haldane, in Proceedings of thee 16th Taniguchi Symposium, Japan, 1993, eds. A.Okiji and N.Kawakami, Springer-Verlag, 1994.

[10] Z.N.C.Ha, IASSNS preprint, 1994.

[11] F.D.M.Haldane and M.R.Zirnbauer, Phys.Rev.Lett.71, 4055 (1993).

[12] B.D.Simons, P.A.Lee, and B.L.Altshuler, Nucl.Phys.B, 1994.

[13] P.J.Forrester, Nucl.Phys.B388, 671 (1992).

[14] G.Moore and N.Read, Nucl.Phys.B360, 362 (1991).

[15] A.Jevicki and B.Sakita, Nucl.Phys.165, 510 (1980); S.R.Das and A.Jevicki, Mod.Phys.Lett. A5, 1639 (1990). 
[16] J.Avan and A.Jevicki, Phys.Lett.B272, 17 (1991).

[17] A. Le Clair, Nucl.Phys.B415, 734 (1994); S.Lukyanov, preprint RU-93-30; C.Efthimiou and A. Le Clair, preprint CLNS 93/1263; D.Bernard and A. Le Clair, preprint CLNS 93/1276.

[18] A.B.Zamolodchikov and Al.B.Zamolodchikov, Ann.Phys.(NY) 120,165 (1979); L.D.Faddeev, Sov.Sci.Rev.Math.Phys.1C, 107 (1980).

[19] J.Avan and A.Jevicki, Phys.Lett.B266, 35 (1991); K.Hikami and M.Wadati, J.Phys.Soc.Jpn., 62, 3857 (1993); preprint UT/W93-021.

[20] Y.Matsuo, preprint UT-661 (1993); H.Awata, M.Fukuma, S.Odake, and Y.Quano, preprint YITP/K-1049 (1993). 